\title{
Ductile damage material parameters identification: Numerical investigation
}

\author{
E. Roux and P.O. Bouchard \\ CEMEF - Centre for material forming, MINES ParisTech, \\ CNRS UMR 7635, Sophia Antipolis, France
}

\begin{abstract}
Calibration of damage parameters is an important issue for the use of damage laws, and particularly for industrial manufacturing processes. This paper deals with an adapted procedure to identify Lemaitre damage parameters using tensile tests. An adapted objective function is built, and Efficient Global Optimisation (EGO) algorithm is chosen to solve the minimisation problem. This procedure is investigated by constructing a landscape of the objective function. This global sensitivity analysis enables to compare different solutions. The sensitivity analysis exhibits some pathological identification issues: multiple extrema and weak gradient. This sensitivity analysis shows some limitations of identification using load-displacement curves: In particularly the non-uniqueness of the set of parameters. The identification procedure is finally validated for an HSS S355MC steel material used in industrial joining application.
\end{abstract}

Keywords: Parameters identification, inverse analysis, global optimisation, sensitivity analysis, ductile damage, global measurement.

\section{Introduction}

An actual industrial issue is the study of material ductility for complex forming operations. The prediction of structural strength of manufactured part requires to couple a forming process and a structural analysis within a numerical virtual chain. To use this virtual chain simulation, material properties must be known. In the cases of mechanical joining processes such as riveting or clinching, materials are submitted to large plastic deformation. The mechanical strength of these joining points is related to the yield and damage history of materials during the joining process [1]. To predict the assembly final mechanical strength, reliable damage parameters are important. 
In this paper we present a methodology to identify material parameters using inverse analysis. Our study focuses on the Lemaitre damage model. To identify Lemaitre damage parameters, local or global measurements can be used. Using global data allows performing non standardized test; for example, a punch test can be exploited [2]. These measures, local or global, are then used in an inverse analysis procedure. Our study aims at working on global measurements resulting from tensile tests. First, tensile test modelling and the Lemaitre damage model are briefly described in order to construct an adapted objective function. Second, a sensitivity analysis is performed on Lemaitre damage parameters. Finally, an adapted inverse analysis procedure is presented. This procedure enables to obtain accurate results for an high strength steel grade.

\section{Direct model}

First steps to build an inverse analysis procedure are to define the direct model, the experimental test and the objective function. The goal of the objective function is to evaluate differences between numerical and experiment results. Once these three points defined, the objective is to match numerical results on experimental results. This correlation is achieved by modifying material parameters using an optimisation algorithm. This global inverse analysis procedure is presented in figure 1.

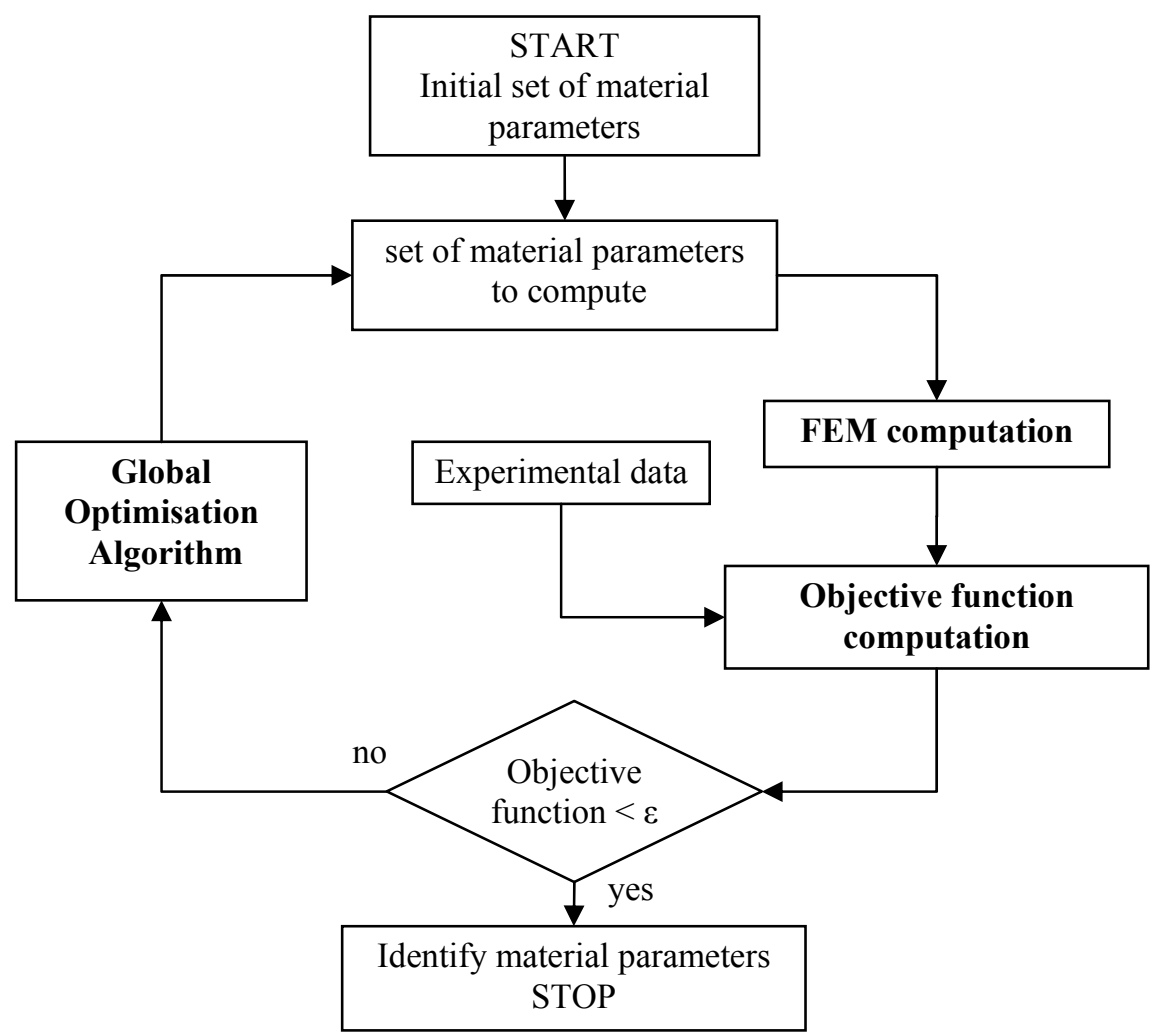

Figure 1: Flow-chart of the inverse method 


\subsection{The Lemaitre damage model}

An elastic-plastic law coupled with a ductile damage model is chosen to describe the mechanical behaviour of the S355MC steel grade studies in this paper. A brief description of the elastic-plastic behaviour and ductile Lemaitre damage model is given here.

Hardening law:

$$
\sigma_{0}=\sigma_{s}+K\left(\overline{\varepsilon_{0}}+\bar{\varepsilon}\right)^{n}
$$

Evolution damage law:

$$
\dot{w}=\left\{\begin{array}{c}
0 \quad \text { if } \quad \bar{\varepsilon}<\bar{\varepsilon}_{d} \\
\frac{\lambda^{p l}}{1-w}\left(\frac{-Y}{S_{0}}\right)^{b} \quad \text { if } \quad \bar{\varepsilon} \geq \bar{\varepsilon}_{d}
\end{array}\right.
$$

The equivalent stress $\sigma_{0}$ is described by a hardening law equation (1), with $\sigma_{s}$ yield stress, $K$ consistency, $\bar{\varepsilon}_{0}$ a regularization parameter, $\bar{\varepsilon}$ the equivalent plastic strain, and $n$ the hardening exponent. The evolution law for the damage parameter $w$ is given by equation (2), $\bar{\varepsilon}_{d}$ is the plastic strain threshold for damage growth, $\lambda^{p l}$ the plastic multiplier, $Y$ the strain energy release rate, $S_{0}$ and $b$ are material damage parameters. More details about this model are given in [4]. The mechanical behaviour is coupled with the damage parameter by computing an effective stress $\widetilde{\sigma}$, as shown in equation (3). A "weak" coupling is used, meaning that value of the damage parameter is updated at end of each increment.

$$
\widetilde{\sigma}=\frac{\sigma}{1-w}
$$

The damage value $w$ gives information on the material health. $w$ equal zero for an undamaged material and grows toward 1 which is reached for complete fracture. The objective of the identification is to determine the parameters values for the damage law. In this paper, we focus on the identification of three damage parameters $\bar{\varepsilon}_{d}, S_{0}$ and $b$.

\subsection{Finite elements modelling}

As said previously, the objective of the inverse analysis is to find a set of damage parameters for which the numerical and experimental observables are as close as 
possible. The first condition of success is to build a numerical modelling representative of the experimental test. The tensile test is chosen here.

Dimensions and shape of the specimen are given in figure $2 \mathrm{a},\left(\mathrm{L}_{0}=61 \mathrm{~mm}\right.$, $\mathrm{D}=20 \mathrm{~mm}$ ). The specimen's thickness is equal to $5.78 \mathrm{~mm}$. $\mathrm{L}_{0}$ is the initial size of the extensometer area. The test is carried out on a classical tensile test machine; the load-displacement curve is recorded (figure 3 ) with an extensometer.

This tensile test is modelling using the CIMLib ${ }^{\circledR}$ library. CIMLib ${ }^{\circledR}$ is a parallel finite element library developed at Cemef. It is based on a mixed velocity/pressure formulation. More details about damage implementation in a mixed velocity/pressure formulation are given in [3].

For the numerical tensile test, a displacement is prescribed at the specimen ends. But the observable measurement is the measurement given by the extensometer, so a numerical extensometer must be used (figure $2 b$ ).

Due to loss of symmetry at end of the test, only one symmetry plan has been used, as shown in figure $2 b$.

(a)

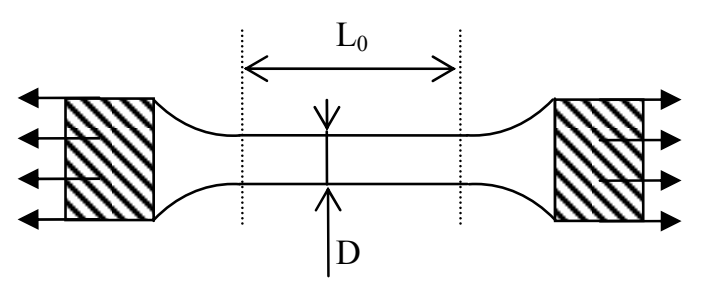

(b)

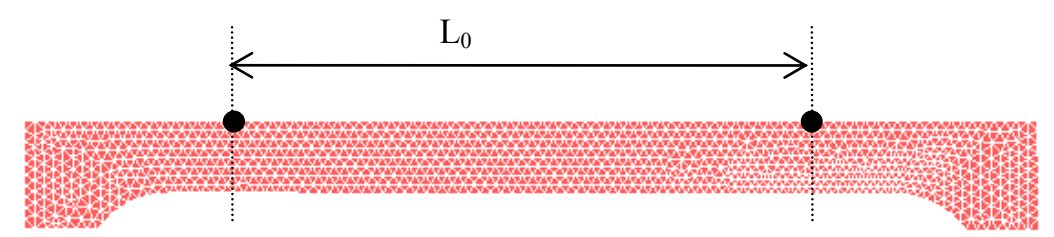

Figure 2: (a) tensile test specimen, (b) finite element modelling (black dots represent captor used for numerical extensometer)

\subsection{Construction of an adapted objective function}

To evaluate the difference between experimental and numerical results we must compute an adapted objective function. We use a formulation based on a least square equation. The discrete formulation of the objective function $\varphi$ is given by equation (4). $y^{\exp }$ are experimental values and $y^{\text {num }}$ are numerical values. The objective function is normalized by the integral of the experimental result. 


$$
\varphi=\frac{\sum_{i}\left[\left(y_{i}^{\exp }-y_{i}^{\text {num }}\right)^{2}\left(x_{i}-x_{i-1}\right)\right]}{\sum_{i}\left[\left(y_{i}^{\exp }\right)^{2}\left(x_{i}-x_{i-1}\right)\right]}
$$

Some precaution must be taken in order to catch the softening part of the loaddisplacement curve. Otherwise the objective function can be non-smooth, which is problematic regarding the optimisation procedure.



(a)

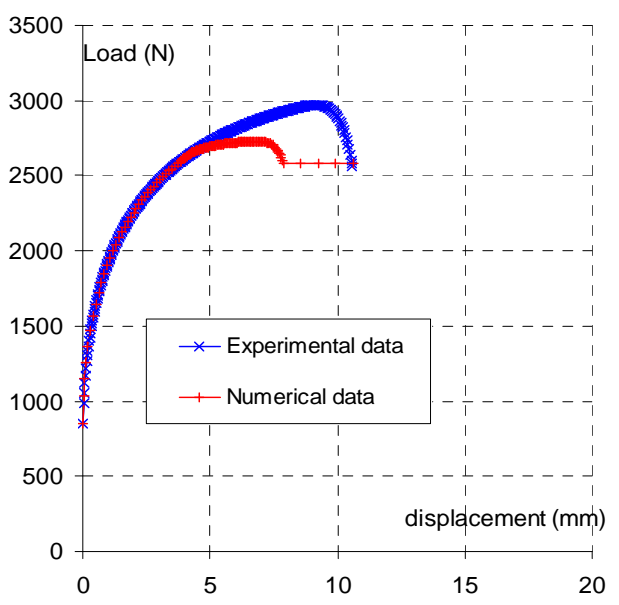

(b)

Figure 3: tensile test load-displacement curve, (a) numerical fracture appears for a larger displacement value than for the experimental curve, (b) numerical fracture appears for a smaller displacement than the experimental fracture displacement

Our first approach was to compute the objective function with the available data. $\varphi$ (equation (5)) is evaluate between $x=0$ and $x=\min \left(x_{\exp }^{f d}, x_{n u m}^{f d}\right) . \quad x_{\exp }^{f d}$ is the experimental fracture displacement and $x_{n u m}^{f d}$ is the numerical fracture displacement. But this formulation leads to a non-smooth objective function as shown in figure 4a. Besides, in some cases, the objective function tends towards zero even if the two curves are different. This case appears when the softening part is very short, so that the objective function is computed only on the hardening part. The identified parameters are therefore wrong.

To solve this problem of wrong objective function, we present here a new objective function. Two particular cases have to be deal with. On the one hand, when the numerical fracture appears for a larger value than the experimental one (figure 3a). And on the other hand when the numerical fracture displacement appears for a smaller displacement than the experimental one (figure 3b). The objective 
function $\varphi$, defined in equation (4), comes from an integral formulation (equation (5)). To handle both particular cases, specific integration domain must be defined.

$$
\varphi=\frac{\int\left(y^{\exp }-y^{\text {num }}\right)^{2} d x}{\int\left(y^{\exp }\right)^{2} d x}
$$

First case: experimental data are completed with one new point defined as follows. The breaking load remains the same whereas the displacement value is chosen to be much higher than the fracture displacement (figure 3a, blue line).

Second case: numerical data are completed by new points defined as follows. Numerical loads remain equal to the numerical fracture load, whereas the displacement value grows from the numerical fracture displacement to the experimental fracture displacement (figure $3 b$, red line).

This adapted formulation gives a smooth and more reliable objective function. The difference between the two formulations is shown in figure 4. The adapted objective function (figure 4b) is smoother than the first one (figure 4a). It makes the optimisation procedure easier.

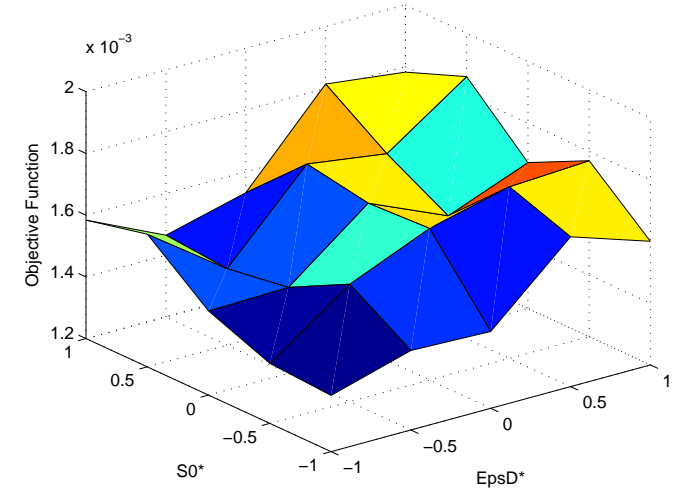

(a)

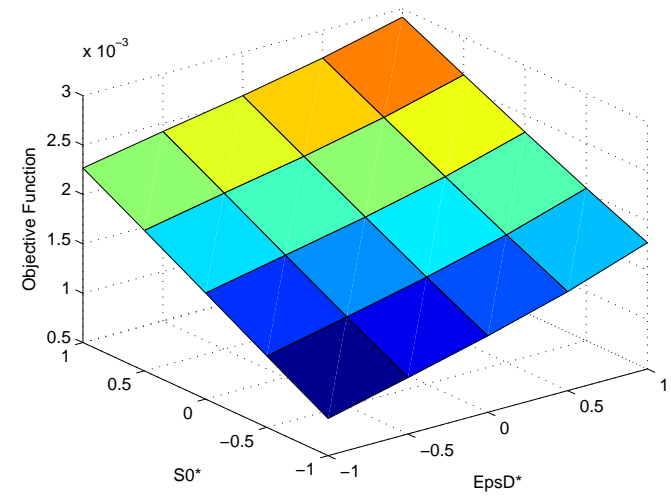

(b)

Figure 4: focus on the objective function, (a) first objective function, (b) adapted objective function

\section{Landscape of the objective function}

To illustrate the methodology, the sensitivity analysis focuses only on 2 parameters: $\overline{\mathcal{E}}_{d}$ and $S_{0}$. The numerical load-displacement curve is compared to a "virtual" experimental curve generated using the parameters presented in table 1. 121 
simulations are preformed here to build a landscape view of the objective function (figure 4).

\begin{tabular}{|c|c|}
\hline Material Parameters & Value \\
\hline$K$ & $430 \mathrm{MPa}$ \\
\hline$\sigma_{s}$ & $46 \mathrm{MPa}$ \\
\hline$n$ & 0.34 \\
\hline $\bar{\varepsilon}_{d}$ & 0.16 \\
\hline$S_{0}$ & 0.7 \\
\hline$b$ & 1 \\
\hline
\end{tabular}

Table1: material parameters for the "virtual" tensile test

\begin{tabular}{|c|c|c|}
\hline Set of parameters & $\overline{\mathcal{\varepsilon}}_{d}$ & $S_{0}$ \\
\hline$s 1$ & 0.16 & 0.7 \\
\hline $\mathrm{s} 2$ & 0.175 & 0.2 \\
\hline
\end{tabular}

Table2: optimal set of parameters

This view gives interesting information to calibrate the inverse analysis procedure. Figure 5 shows that the minimisation problem has multiple optima ( 2 in this case), and weak gradient area. Optimisation algorithm must be robust enough to deal with multiple extrema and weak gradient.

The sensitivity analysis gives other interesting information: multiple set of parameters can give a valid solution regarding load displacement-curve ( 2 in this case). The first optimum is located at $s 1$ which are the right parameters (table 2). The second optimum is located at $s 2$. The limit of parameters identification base on the load-displacement curve appears. The information contained in loaddisplacement curve is too poor to identify damage parameters. This is due in particular to the fact that the softening part of load-displacement curve is due both to damage growth and to necking. A second observable would be necessary to reduce the number of solution to one.




Figure 5: objective function landscape

To reduce the number of solutions, a new observable is computed: necking. Indeed, as said previously, the softening of the tensile load is due both to damage growth and to necking. A new objective function is computed in the same way as for the load-displacement curve (equation 4), but with accounting for necking-displacement experimental and numerical curves. The landscape is presented in figure 6 . Using this new objective function, the second solution obtained previously (parameter set $s 2$ ) can be eliminated since it is not a minimum of the necking objective function landscape. The necking objective function has for set $s 1$ an optimum, and a large area $\left(0 \leq \bar{\varepsilon}_{d} \leq 0.14,0.2 \leq S_{0} \leq 0.8\right)$ where the objective function is weak. But has shown by the objective function based on load-displacement curve this area is not a minimum. So necking-displacement can not be used alone, but it is complementary to load-displacement observable. This first numerical test shows that adding one observable is a good way to reduce the number of solutions.

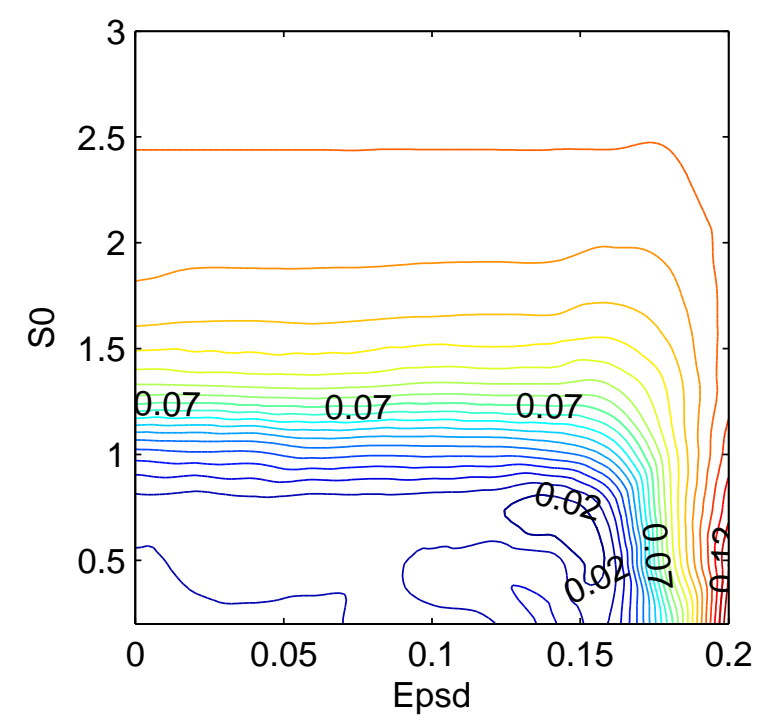

Figure 6: necking objective function landscape

\section{Identification by inverse analysis}

\subsection{An adapted optimisation method}

Identification of damage parameters requires the use of an advanced optimisation procedure.TheEfficient Global Optimisation (EGO) algorithm is used [5]. This algorithm is based on an iterative update kriging meta-model. We use here a metamodel assisted method in order to reduce the computation time.

Figure 7 shows optimisation results obtained using the EGO algorithm. An optimal solution is found after 40 objective function exact evaluations. The 2 local minima 
are explored. Areas of weak gradient are mapped with multiple points. This test prove that the chosen algorithm is adapted to our identification problem.

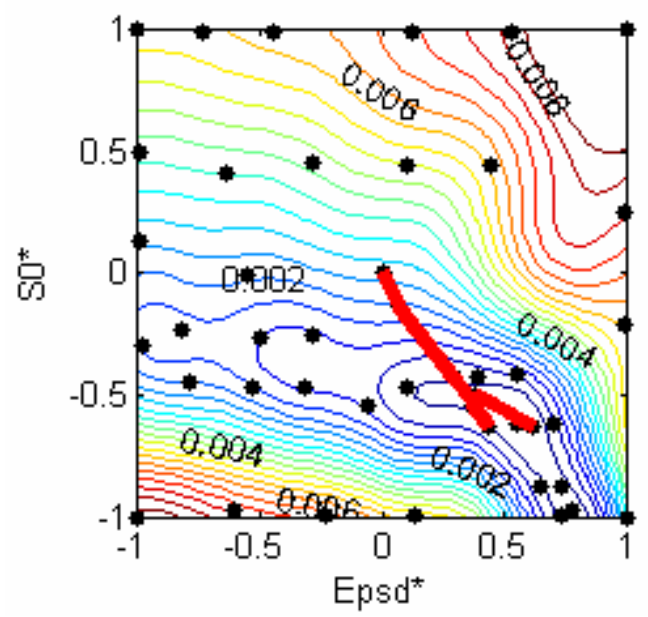

Figure 7: minimisation of the objective function (meta-model contour, black dot: master points, red line: optimum way)

\subsection{Application}

This calibration enables to identify damage parameters from real experimental results. Here a HSS S355MC steel is tested. The identification procedure gives accurate results (figure 8). Identified damage parameters are $S_{0}=0.71$ and $\bar{\varepsilon}_{d}=0.16(b$ is set to 1); the final objective function is equal to $3 \mathrm{e}-4$.

The only difference, appearing at the beginning of the softening part comes from the incapacity of the chosen damage model to represent the initial softening, whatever the damage parameters used. In such cases, it would be recommended to modify the formulation of damage growth in the model. However, such as a difference is of the same order than uncertainty measurement or material variability. The solution is thus acceptable. Finally, as show in the previous part, there is no proof of the result uniqueness.

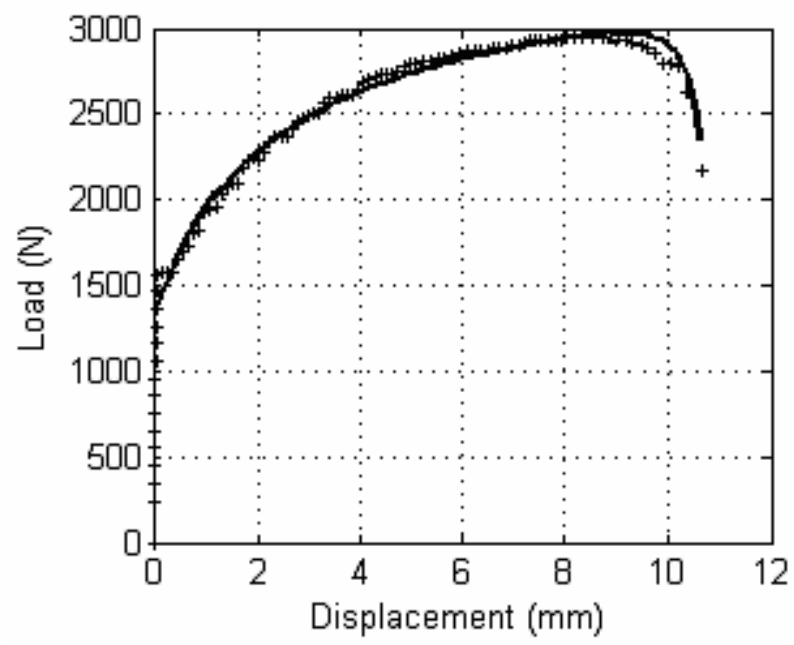


Figure 8: load-displacement curve identification result (cross: experimental data, line: identify solution)

\section{Conclusion}

In this paper, an objective function adapted to mechanical softening behaviour identification has been presented. This adapted objective function allows obtaining a smooth and reliable optimisation problem.

Then, a global sensitivity analysis has been performed on two damage parameters. The major observation done by this sensitivity analysis is that the minimisation problem has multiple extrema and weak gradient area. This means that information contained in the load-displacement curve are not rich enough to get a unique solution. This issue can be tackled by using enhanced experimental data. The global softening behaviour of load-displacement curves is due both to damage and necking. Measuring necking evolution during the tensile test enriches the objective function and reduces the number of solutions. Another technique would be to use field measurement in the deformed area, in order to get more local and accurate information.

\section{Acknowledgements}

CeTIM Industrial and Mechanical Technical Centre is gratefully acknowledgement for their financial support within this study.

\section{References}

[1] P.O. Bouchard, T. Laurent, L. Tollier, J. Mater, "Numerical modelling of selfpierce riveting from riveting process modelling down to structural analysis". Process. Technol. 202(1-3), pp. 290-300, 2008.

[2] M. Abendroth, M. Kuna, "Identification of ductile damage and fracture parameters from the small punch test using neural networks", Engineering fracture mechanics, Springer, 2006.

[3] R. EL Khaoulani, "Prédiction fiable de l'endommagement ductile par la méthode des éléments finis mixte : endommagement non local et adaptation de maillage", PhD Mines ParisTech, 2010.

[4] J. Lemaitre, A course on damage mechanics, Springer-Verlag (second edition), 1996.

[5] D. R. Jones, M. Schonlau, W. J. Welch, "Efficient Global Optimization of expensive black-box functions", Journal of global optimization 13, Springer, pp.455-492, 1998. 